\title{
Transport hydraulique de graviers et galets en conduite
}

\author{
The hydraulic transportation \\ of gravel and pebbles in pipes \\ PAR ROBHRT DURAND \\ INGÉNEUR aUx ETablssements Nernple
}

English synopsis, p. 578.

Nous avons étudié, au Laboratoire Dauphinois d'Hydraulique, depuis 1948 , les conditions de refoulement hydraulique en conduite horizontale de différents matériaux. Nous exposerons ici les recherches effectuées sur des graviers et des galets à l'occasion de l'étude du circuit de refoulement d'une drague stationnaire à godets refouleuse, construite par les Ateliers et Chantiers de Bretagne pour la Compagnie Nationale du Rhône. Cette dragule, destinée à travailler dans le périmètre de Donzère, doit refouler des déblais pouvant avoir jusqu'à $100 \mathrm{~mm}$ de diamètre.

La première partie de notre étude a porté sur le puits de reprise des déblais pour lequel une solution originale avait été proposée par les A.C.B. Cette solution a donne toite satisfaction et son étude fera l'objet d'une publication ultérieure.

La seconde partie a porté sur les conditions de refoulement proprement dites. La conduite prévue pour la drague avait pour diamètre $330 \mathrm{~mm}$ et pour longueur $400 \mathrm{~m}$. La concentration envisagéc élait de $8.75 \%$ en volume apparent, soit $140 \mathrm{gr} / 1$.

L'échelle du modèle a été choisic de l'ordre du $1 / 3$, exactement $1 / 3,17$, car nous avons adopté une tuyauterie de type standard de $104 \mathrm{~mm}$ de diamètre. Les matériaux utilisés pour l'étude provenaient des banes de graviers de Donzère. Leurs dimensions ont été choisies à la mème échelle du tiers. Il est à remarquer que ces matériaux sont les mêmes que cenx ćtudiés par Du Boys dans son article classique publié dans les Annales des Ponts et Chaussées en 1878.

Leur densité absolue moyenne était de 2,65 et leur densité apparente voisine de 1,60.

Les matériaux ont ćté triés grâce à des tamis à maille carrée en cinq catégories comprises entre les limites suivantes (vide de maille) $2,3 \mathrm{~mm}$ $-5,25 \mathrm{~mm}-9,9 \mathrm{~mm}-15,54 \mathrm{~mm}-20 \mathrm{~mm}-$ $25 \mathrm{~mm}$.

Ce triage était nécessaire du fait que notre modèle fonctionnait en circuit fermé. Nous renverrons pour ce point le lecteur à un article publié dans le ${ }^{\circ} 3$ de mai 1951 de la Houille Blanche, ainsi que pour la définition des notions de concentration de transport et de concentration spatiale que nous utiliserons dans la suite de notre exposé.

\section{I. - CONSIDÉRATIONS SUR LES VITESSES DE CHUTE DES ALLUVIONS DU RHONE}

L'essai physique préliminaire essentiel pour l'étude du refoulement en conduite d'une mixture est la détermination de la vitesse de chute de grains isolés dans un fluide au repos en milieu infini.

Un grain de maître couple principal $\mathrm{S}$ et de 
poids apparent $P_{a}$ dans un fluide de masse spécifique: prend une vitesse de chute limite W.

$$
P_{n}=C_{a}: S-\frac{W^{2}}{2}
$$

Pour une sphère, on connait bien la variation du coefficient de trainéc $C_{x}$ défini par l'équation précédente en fonction du nombre de REyNoLds hasé sur le diamètre de la sphère (voir figs. 1). Pour des nombres de Rexnolds allant jusqu’à 1 , on a :

$$
\mathrm{C}_{v}=\frac{24}{\mathrm{R}}
$$

ce qui correspond au régime laminaire. Pour des nombres de REynolos compris entre $10^{\circ}$ et $10^{\circ}$, $C_{x}$ reste sensiblement constant, ce qui correspond au régine turbulent.

Parallèlement, la courbe des vitesses de chute en fonction du diamètre présente également deux parties distinctes. Pour des grains de diametro inférieur à $0,15 \mathrm{~mm}$ environ (grains de densité 2,65 en chute libre dans l'eau), on a la loi de STokes de la forme (fig. 2) :

$$
\mathrm{C}_{x}=\frac{24}{\mathrm{R}} \quad \mathrm{W}=\mathrm{K} d^{\prime \prime}
$$

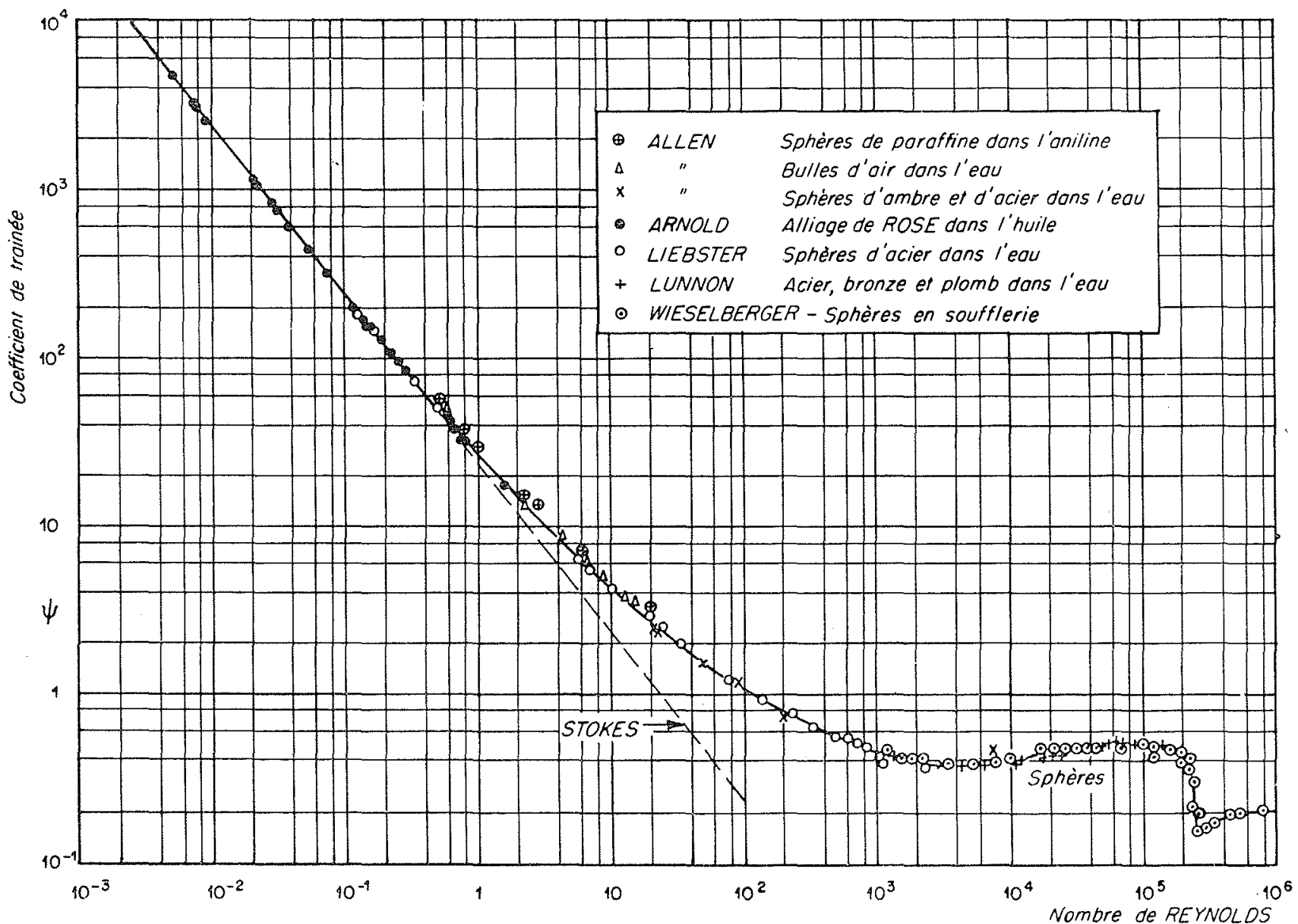

Fir, 1. - Variation du coefficient de trainéc pour des spheres en fonction du nombre de Rryouns.

Cette classe correspond aux argiles, limons et sables fins.

Pour des grains de diamètre supérieur à $1,5 \mathrm{~mm}$ environ, on a une loi définie par RitrisGER comme étant de la forme :

$$
\mathrm{C}_{, r}=\mathrm{C}_{0} \quad \mathrm{~W}=\mathrm{K} \sqrt{d}
$$

Il s'agil de déblais désignés communément sous le nom de graviers et galets.
Entre 0,1 et $1,5 \mathrm{~mm}$, on a une loi de transition qui correspond à :

$$
\mathrm{C}_{x}=\frac{24}{\mathrm{R}}+\mathrm{C}_{0}
$$

C'est sensiblement le domaine des sables grossiers.

Nous voyons done que, de ce point de vue, les matériaux de densité 2,65 (densité la plus fré- 


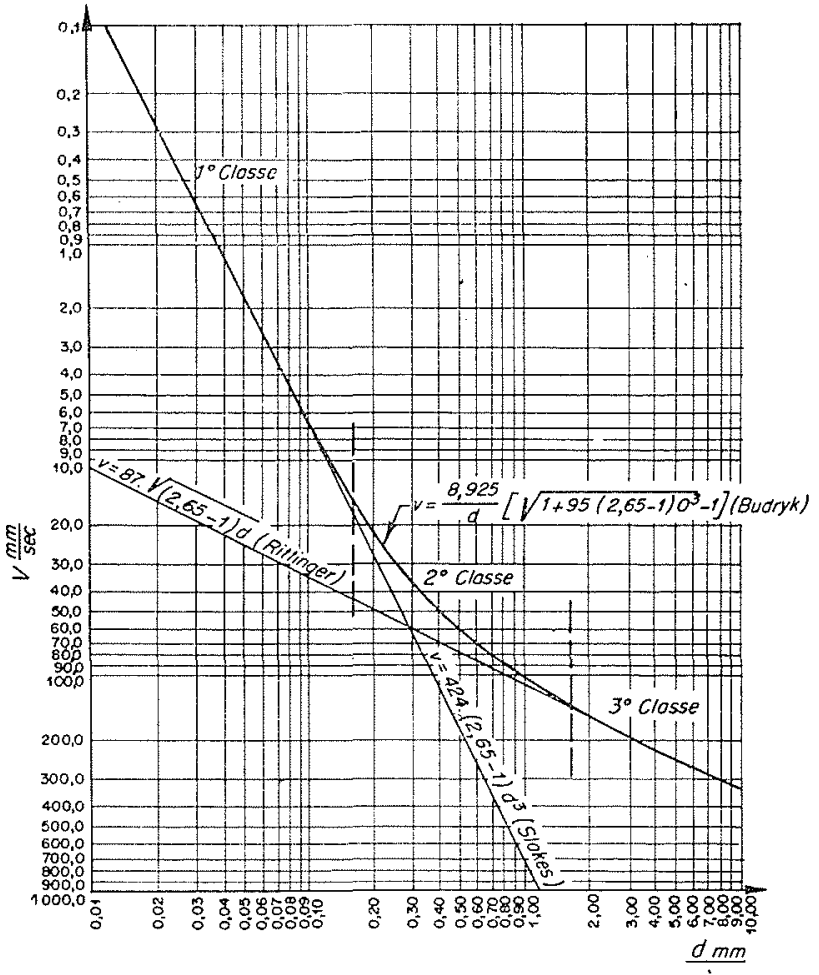

FIg. 2. - Vitesse de chute dans l'eau en fonction du diametre nominal pour des grains de quartz de densite 2,65 (d'après Budryck, essais de Richards). (quente des alluvions naturelles) se partagent on trois classes en fonction du diametre, ces classes étant séparées approximativement par les valeur's 0,15 et $1,5 \mathrm{~mm}$. Ce mode de classement a déjà dé signalé par de nombreux auteurs et en parliculier par Sir Claude INaLis. Il est bien évident d'ailleurs que les valeurs numériques critiques indiquées ne correspondent pas à un chansement brutal de propriétés.

Les considérations précédentes étaient valables dans le cas des sphères. Or, la forme des alluvions du Rhône s'écarte en moyenne notablement de celle de la sphère. Il fallait done voir dans quelle mesure cette forme particulière influençait la vitesse de chute. De toute façon, pour permettre des recoupements ultérieurs avec des essais sur la drague réelle, il fallait définir et chiffrer la forme des galets étudiés.

Nous avons choisi comme critère le coefficient volumétrique $C$ défini par les normes francaises. C'est le rapport du volume V du caillou (déduit d'une mesure de poids, la masse spécifique étant connue) au volume de la sphère ayant pour diamètre la plus grande dimension L du caillou :

$$
\mathrm{C}=\frac{\mathrm{V}}{\frac{\pi}{6} \mathrm{~L}^{3}}
$$

Le coefficient volumétrique $\mathrm{C}_{m}$ d'un mélange est égal au rapport du volume d'un certain nom-
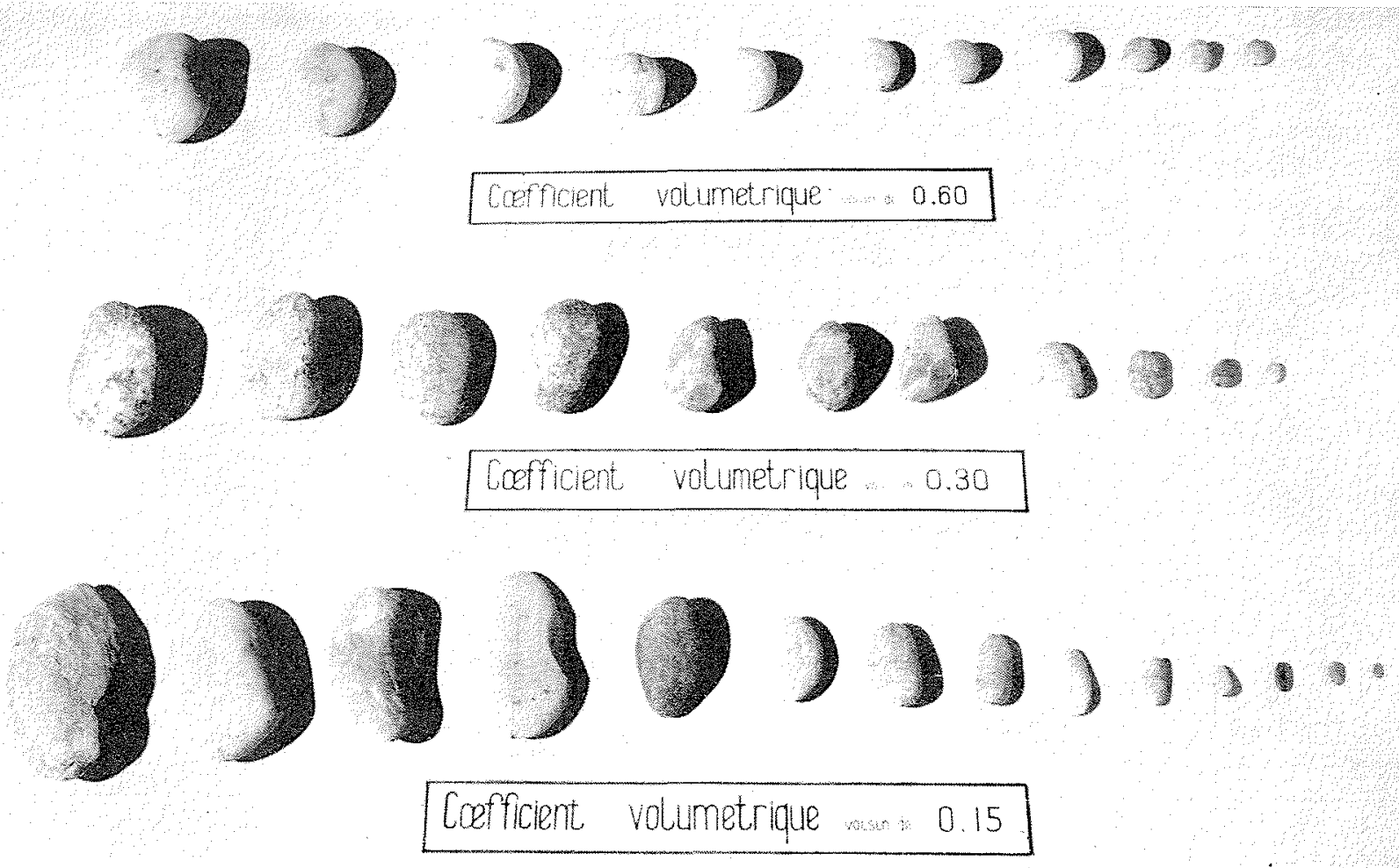

Fra. 3. - Galets du Rhône classés en fonction de la valeur du coefficient volumétrique. 
bre de grains a la somme des $\frac{\pi}{6}$ de chacun d'eux.

Les coefficients volumétriques moyens mesurés sont en général voisins de 0,3 . L'ensemble de nos mesures a porté sur environ 750 galets.

Remarquons que la valeur moyenne de 0,3 semble normale. Pour le gravillon de la Scine, Favry indique la valeur de 0,22 et pour la Moselle la valeur exceptionnelle de 0,4 .

A priori, l'emploi du seul coefficient volumétrique peut sembler insuffisant pour définir le comportement d'un grain dans un écoulement. Mais les galets ayant été roulés par un fleuve dont le régime est relativement uniforme dans cette région, il doit exister, du fait de ce façonnement naturel, une relation implicite entre les divers coefficients de forme qui permettent de définir un grain.

Dans le but de vérifier ce point, nous avons trié à la main un certain nombre de galets. Ces galets ont été choisis de telle facon que leur coefficient volumétrique soit voisin de l'une des trois valeurs particulières suivantes : $0,6-0,3$ - 0,2. Chacun d'eux a été caractérisé en outre

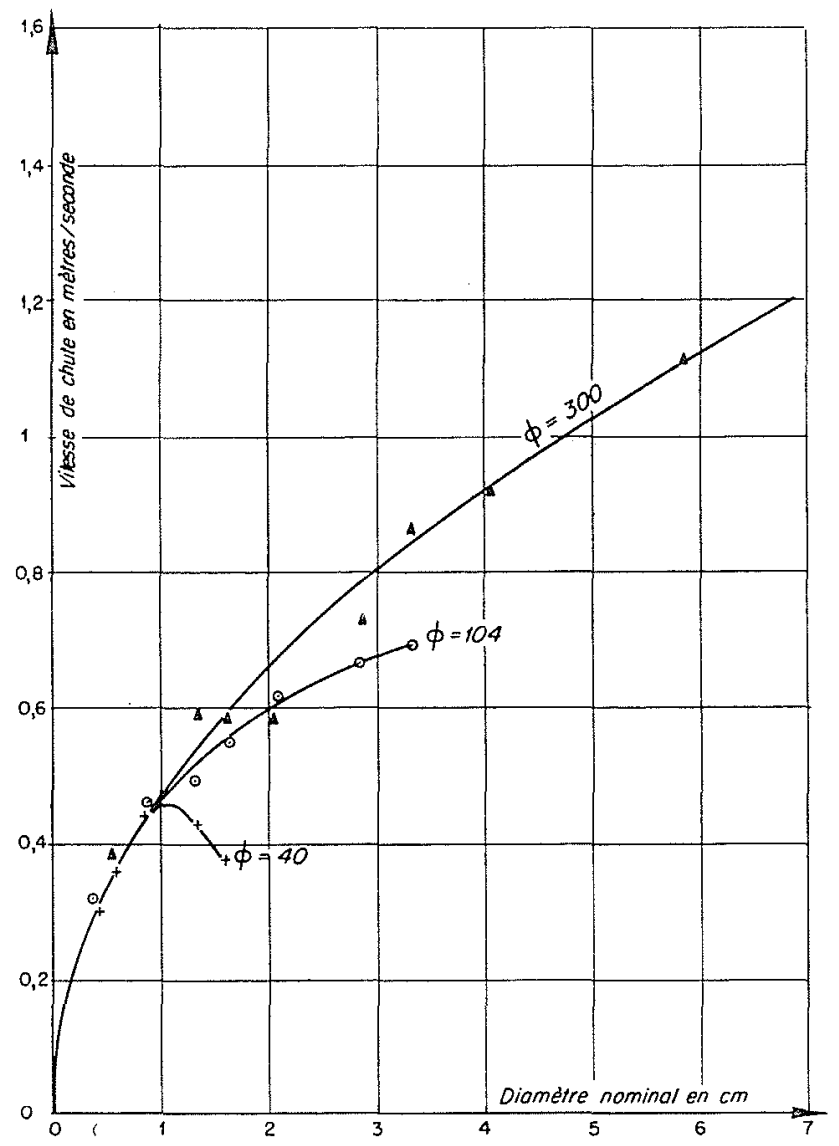

Fici, 4. - Variation de la vitesse de chute en fonction du diamètre nominal des galets. Coefficient volumètrique voisin de 0,30. Essais dans trois conduites de $\varnothing 40$, 104,300 . par son diametre nominal $d n$, e'est-à-dire par le diametre de la sphère de même volume.

Si L est la plus grande dimension du galet entre plans paralleles, $L^{\prime}$ la plus grande dimension normale à la direction précédente et $\mathrm{I}^{\prime \prime}$ la plus grande dimension normale aux deux précédentes, on peut appeler module d'allongement le rapport $\frac{\mathrm{L}}{\mathrm{L}^{\prime}}$ et module d'aplatissement le rapport $\frac{L^{\prime}}{L^{\prime \prime}}$. En moyenne, ces modules sont égaux pour un caillou du Rhône donné, quel que soil son diamètre nominal et son coefficient volumétrique. A titre de contrôle, nous avons effectué les mêmes mesures sur les galets du Drac à Grenoble et nous n'avons pu dégager aucune relation entre ces deux modules. Il existe donc bien une certaine uniformité particulière dans la forme des galets du Rhône étudiés (voir fig. 3).

Nous avons alors réalisé des essais de vitesse de chute pour les différents galets particuliers choisis. Ces essais ont été effectués de facon assez sommaire dans un esprit pratique, c'est-à-dire sans s'attacher à une trop grande précision.

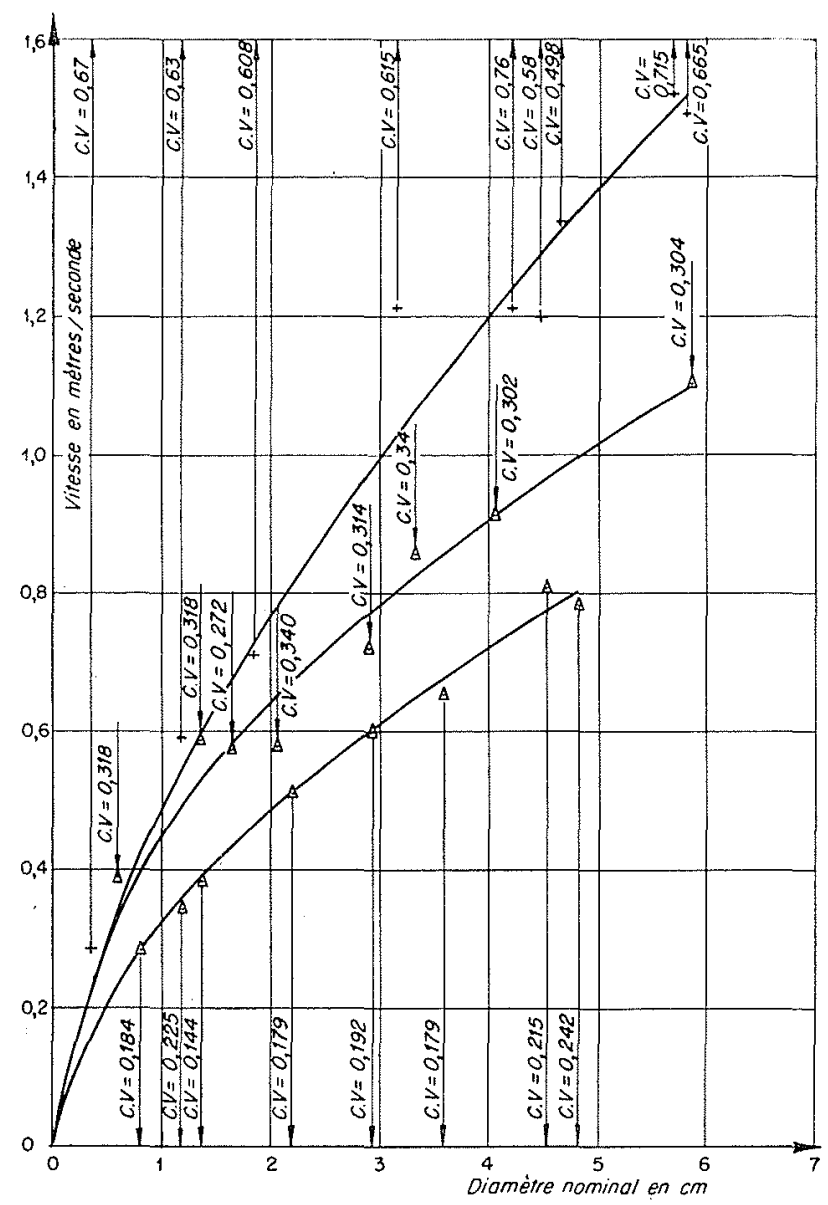

Fili, 5. Vitesse de chute en fonction du diametre nominal pour res galets du Rhone de coefficient volumétrique voisin de $0,60,0,30,0,20$. (Diamètre de la conduite d'essai : $300 \mathrm{~mm}$.) 
'Trois tuyateries verticales de $7 \mathrm{~m}$ de hauteur' ont été dressées. Elles avaient respectivement pour diametre $40 \mathrm{~mm}$ - $104 \mathrm{~mm}-300 \mathrm{~mm}$. A trois metres du sommet, se trouvaient des regards vitrés et le bas des conduites étail obturé par un fond transparent en plexiglas. Ces conduites étant remplies d'eau, le galet était lâché successivement au sommet de chacune des conduites. Un premier observateur déclanchait un chronomètre électrique au passage du galet devant le premier regard. Un second observateur arrêtait le chronomètre lorsque le galet atteignait le fond. Nous avons ainsi pu tracer les courbes des figures 4 et 5 . La figure 4 montre l'inftuence des parois sur la vitesse de chute pour des galets de coefficient volumétrique voisin de 0,3 . On voit que cette influence devient nettement sensible lorsque le diametre du galet est plus grand que le quart du diametre de la conduite.

Ia figure 5 montre la valeur de la vitesse de chute en fonction du diamètre nominal du grain (diamètre de la sphère de même volume) pour les essais dans la conduite du diamètre 300 . Les points d'essais sont cotés suivant la valeur exacte du coefficient volumétrique. On voit que les points s'ordonnent assez bien autour de courbes moyennes pour chaque valeur moyenne du coefficient C. De même, si l'on exprime le coefficient de traince en fonction du nombre de RErNolds, on constate qu'il demeure sensiblement constant pour chaque catégorie.

Ce résultat montre que le coefficient volumétrique suffit pour les galets du Rhône à caractériser la forme, du point de vue comportement des galets dans l'eau, et que leur vitesse de chute reste de la forme :

$$
\mathrm{W}=\mathrm{K} \sqrt{d}
$$

Par suite, la classification en trois catégories, établic pour les sphères, leur demeure applicable.

\section{II. - DESCRIPTION DU REGIME DE SALTATION}

Nous décrirons tout d'abord ce que nous a permis d'observer un élément vitré intercalé sur le circuit de refoulement de $104 \mathrm{~mm}$ de diamètre de notre modèle.

Considérons dans la conduite horizontale un dépôt uniforme de graviers de diamètre compris entre 2,3 et $5,25 \mathrm{~mm}$ (vide de maille).

Augmentons progressivement la vitesse de l'eau à partir de zéro. Au-delà diune certaine vitesse $V_{1}$, de l'ordre de $0,5 \mathrm{~m} / \mathrm{s}$, les graviers situés en surface du dépôt commencent à s'agiter, puis basculent les uns sur les autres. Tous les grains ne se mettent pas en mouvement simultanément car ils n'occupent pas initialement la mème position les uns par rapport aux autres. En augmentant un peu la vitesse de l'eau, ces différences deviennent insensibles et le nombre de graviers mis en mouvement s'aceroit régulièrement. Dès que la vitesse moyenne est légèrement supérieure à la valeur nécessaire pour assurer le basculement des grains, ces derniers se mettent a progresser par bonds, ce qui justifie le terme classique de saltation. Ce régime de transport a été souvent décrit, el plus particulierement par M. BAGNoLD dans son livre sur le transport des sables par le vent. Alor's qu'à la lecture de la plupart des ouvrages traitant de la saltation on peut croire qu'il s'agit là d'un phénomène transitoire, M. BAGNOLD insiste sur le fait que e'est pour les sables dans l'air un mode de transport caractéristique qui se maintient pour tontes les vitesses pour lesquelles le matériau est nettement entraîné. C'est ce que nous avons pu également constater pour les graviers et galets dans l'eau, mème pour des vitesses de l'ordre de $4,5 \mathrm{~m} / \mathrm{s}$. Le régime de saltation n'apparaît done pas comme étant un mode transitoire de transport, mais comme fondamental pour les gros matériaux.

On distingue d'ailleurs très nettement, par observation directe a travers un regard vitré, si les matériaux sont en régime de suspension on de saltation, même aux vitesses trop grandes pour que l'œil puisse suivre individuellement les grains. En régime de suspension, les particules visualisent les tourbillons de l'écoulement, ce qui produit une impression de grouillement absolument désordonné. En régime de saltation, on distingue des stries qui oscillent en donnant une impression de scintillement tout en restant en moyenne nettement dirigées suivant la direction de l'écoulement.

Essayons maintenant de domner une description plus détaillée du mécanisme de saltation. Pour un matériau homogène, à des vitesses peu supérieures à la vitesse d'entrainement, le gravier qui vient d'effectuer un bond peut, au moment où il rejoint le dépôt, soit ricocher sur un grain immobile pour décrire une nouvelle trajectoire, soit ereuser dans le dépôt une sorte de petit cratere en éjectant d'autres grains auxquels il restitue une partie de l'énergie cinćtique qui lni avait été communiquée par le fluide. $A$ de plus grandes vitesses, l'énergie acquise par 
les grains est suffisante pour leur permettre à la fois de ricocher et de mettre en mouvement les graviers qu'ils viennent de heurter. On arrive ainsi à avoir en dessous des couches supéricures progressant par bonds, une ou plusicurs couches progressant par glissement. La vitesse de chacune de ces couches en glissement décroît à mesure que l'on se rapproche de la partie du dépôt restée immobile. Leur nombre augmente avec la vitesse moyenne de l'eau. On retrouve là lc schéma classique donné par Du Boys à propos, rappelons-le, des mèmes matériaux que ceux que nous avons observés. Mais alor's que cet auteur considère que ce charriage est dû directement à l'effort tangentiel à la paroi exercé par l'écoulement, il se produit en fait par l'intermédiaire des grains en régime de saltation. Ces derniers progressent beaucoup plus rapidement que les couches en glissement et le débit solide correspondant au charriage ("surface creep" pour" M. BAGNOLD) ne représente qu'une partie du débit solide total.

A ce phénomène de la saltation, qui est absolument général pour certaines catégories de matériaux que nous préciserons plus loin, peut se superposer le phénomène particulier des dunes.

Si fortuitement un grain de la couche en charriage immédiatement en contact avec le dépôt vient à s'immobiliser, il tendra à arrêter les graviers au même niveau, situés plus à l'amont. Les couches supéricures seront ralenties de proche en proche et tout se passera finalement comme si l'on avait rajouté localement une couche de graviers, ces graviers étant prélevés sur le même débit solide permanent des régions supérieures en saltation. Si le même phénomène se reproduit au mème endroit et plusieurs fois, il peut se former une dune car le rouleau à axe horizontal qui se forme à l'aval tend à la stabiliser. Dans notre installation en circuit fermé, ces dunes finissaient par s'individualiser au point de laisser entre elles des tronçons de conduite vides de gravier's.

Une dune isolce présente alors l'aspect suivant : elle comporte un noyau immobile dont le front est incliné suivant une pente douce sensiblement uniforme. Cette pente augmente légèrement avec la vitesse moyenne de l'eau tant que les dunes demeurent distinctes. A mesure que l'on s'élève le long du talus, du fait de l'accroissement de vitesse résultant de l'obstruction croissante de la conduite, les graviers sont soumis de façon de plus en plus intense au phénomene de saltation. Si la dune est suffisamment importante, le talus amont est suivi d'un palier. A son extrémité aval, la dune présente un front raide à l'arrière duquel se développe un rouleau à axe horizontal. Les conches inférieures en mouvement, qui progressent par glissement, s'éboulent directement suivant un angle voisin de l'angle de talus naturel, et en fait mème un peu supérieur sous l'effet du courant de retour dû au roulcau. Les graviers progressant en saltation peuvent soit atteindre la conduite dans la zone d'action du rouleau, auquel cas ils sont ramenés vers le pied aval de la dune, soit plus loin. Ils continuent alors à progresser, mais leur vilesse de progression sur fond lisse est plus rapide que sur le corps de la dune et ils tendent à rattraper la dune suivante (fig. 6). Suivant les termes de

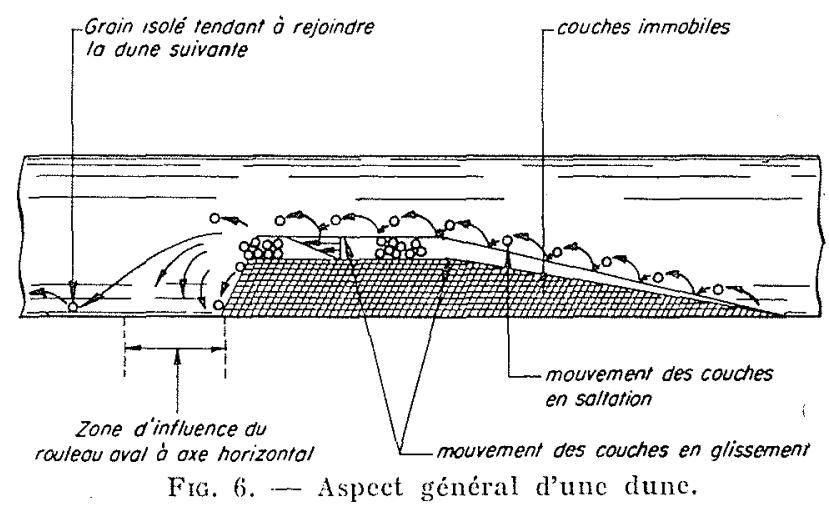

De But (1876), le gravier ou «le grain de sable, poussé par le courant, montre la pente douce du premier talus et arrive au sommet; il roule par son propre poids du haut en bas du talus opposé; là, il demeurera au repos, à l'abri de l'aclion du fluide, et il est recouvert par d'autres grains qui viennent à leur tour... Ces grains de sable ainsi enterrés restent en repos jusqu'à ce que toute la masse qu'ils avaient laissée en arrière ait passé en détail au-dessus d'eux».

La vitesse fictive de progression de la dune est ainsi bien inférieure à la vitesse moyenne de l'eau.

Lorsqu'on augmente cette dernière notablement, le nombre de grains en saltation sur le dessus de la dune s'accroit ainsi que la proportion de grains qui échappent à l'influence du rouleau aval. L'individualité des dunes tend alors à s'atténuer pour ces deux raisons jusqu'au moment où la dernière couche du corps fixe de la dune se met à son tour à glisser. Nous désignerons le type de dune correspondant par l'expression de «dune limite $»$. La vitesse moyenne, dite vitesse critigue, est alors de l'ordre de $2,2 \mathrm{~m} / \mathrm{s}$ pour nos grains de 2,3 et $5,25 \mathrm{~mm}$ de diametre dans une conduite de $\varnothing 104 \mathrm{~mm}$. Ils sont répartis de façon à peu près uniforme le long de la conduite. On a toujours saltation des couches supérieures el glissement des couches inférieures. Si quelques grains de la couche immédiatement en conlact avec la génératrice inféricure viennent à s'immobiliser, les couches superieures s'arrêtent à leur tour, créant une obstruction 
dans la conduite. Les vilesses chant deveres, il apparait à la fois une différence de pression entre les deux faces de l'obstacle et une dépression au sommet (par effet Venturi). L'ensemble, provisoirement immobile, glisse alors d'un bloc en se désagrégeant sous l'effet de la dépression. En augmentant encore un peu la vitesse moyenne, ces instabilités disparaissent et le régime de saltation se conserve.

Il se conserve également pour les graviers plus gros que coux considérés jusqu'à présent, à savoir 5-10, 10-15, 15-20, 20-25 el pour divers mélanges-types étudiés. On peut remarquer que, pour une mème vitesse de l'écoulement, les trajectoires moyennes sont d'aulant plus hautes et plus longues que les galets sont plus gros, dans la mesure bien entendu où la vitesse est suffisante pour les entrainer.

Si nous étudions alors le refoulement d'une mixture à granulométrie étendue, les gros graviers ayant des trajectoires plus tendues échappent plus facilement au rouleau aval. En moyenne, ces grains seront moins souvent immobilisés dans le corps fixe de la dune que les graviers plus fins et auront donc une vitesse moyenne supérieure. Du même coup, un débit solide important circulera constamment entre les dunes et lindividualité de ces derniores lendra à s'atténuer. C'est ce qui explique que te phénomène n'ait pas été signalé neltement à notre connaissance dans la litterature technique. Cependant, G. H. Howarp signale un processus analosue à l'occasion d'une étude portant sur le refoulement dans une conduite de 4 pouces de graviers dénommés "Pea Gravel", de granulométrie comprise entre 0,5 et $7 \mathrm{~mm}$. Il qualifie l'écoulement de saccadé et le caractérise par le mot "jerking ". M. Danel, dans une réponse à l'article de Howard, compare ce mouvement saccadé à un phénomène analogue observable sur les torrents de montagne.

Pour du sable, le transport par saltation joue dans une gamme plus réduite. Pour un régime correspondant à la disparition des dumes, la majorité des grains est alors en suspension et les phénomènes décrits précédemment à propos de la dune limite deviennent insensibles.

Pour les graviers, l'existence de dunes isolées en matériau homogène est évidemment facilitée par la conception en circuit fermé de notre modèle. Le phénomène doit être moins net en circuit ouvert où l'on réalise une alimentation régulière en tête de l'installation.

\section{III. - SIMILITUDE ET RESULTATS D'ESSAIS}

Il semble logique d'essayer de caractériser les ćcoulements de mixture par la valeur du rapport $\frac{W}{V}$, de la vitesse de chute d'une particule isolée en régime uniforme à la vitesse moyenne de l'écoulement.

Parmi les caractéristiques physiques d'un grain bien définies et relativement faciles à déterminer, c'est en effet celle qui semble la mieux appropriée au problème du transport du grain par l'eau.

Son emploi a d'ailleurs déjà été sanctionné par l'expérience puisque le nombre $Z$ de la théorie de Schmidt-Rouse caractérise le degré de mise en suspension des matériaux fins en faisant intervenir le même rapport $\frac{W}{V}$.

$$
Z=\frac{C W}{0,4 V}
$$

arec $\mathrm{C}$, coefficient de CHÉzY.

Cette théorie a été vérifiée pour les canaux aux faibles concentrations. D'après les essais effectués par nous sur des particules fines de suies, l'emploi du nombre $Z$ semble être également jus- tifié en conduite circulaire et pour ces concentrations déjà importantes.

Nous allons examiner de plus près, en explicitant $W$ en fonction des variables usuelles, quelle condition impose l'hypothèse que $\frac{W}{V}$ ou son inverse $\frac{V}{W}$ caractérise l'écoulement d'une mixture.

Pour des particules supposées sphóriques :

$$
\mathrm{W}^{2}=\frac{4}{3} \frac{g d}{\mathrm{C}, r} \stackrel{\rho^{\prime}-o}{?}
$$

Pour les matériaux de la première classe (argiles, limons et sables fins) :

$$
\mathrm{C}=\frac{24}{\mathrm{R}} \text { avec } \mathrm{R}=\frac{\mathrm{Wd}}{\mathrm{y}}
$$

et : $\quad \frac{V}{W}=18 \frac{\rho}{q^{\prime}-q} \frac{V y}{g d^{2}}$

Pour les matériaux de la troisième classe (gra- 
viers et galets) pour lesquels $C_{n}$ est constant, on aura :

$$
\left(\frac{\mathrm{V}}{\mathrm{W}}\right)^{2}=\frac{3 \mathrm{C}_{x}}{4} \frac{\rho}{?^{\prime}-?} \frac{\mathrm{V}^{2}}{g d}
$$

Ainsi pour ces matériaux l'hypothèse suivant laquelle l'écoulement est caractérisé par $\frac{W}{V}$ se ramène, pour des matériaux de densité donnće dans l'eau, à prendre comme paramètre de similitude $\frac{V^{2}}{g d}$, nombre sans dimensions caractéristique de la similitude de Froude.

Le fait que pour de tels matériaux le coefficient de traînée est indépendant du nombre do REyxolds, done de la viscosité, laisse d'ailleurs prévoir la similitude de Froude.

M. Meyrr-Peter a donné depuis 1934 une formule qui vérifie en canal cette similitude à partir de ses propres essais el de ceux de Gilizrrt. Il est à remarquer que tous les matériaux qu'il avait considérés avaient un diamètre supérieur à $3 \mathrm{~mm}$ et appartenaient done bien à la troisième classe au sens où nous l'avons' définie.

Remarquons que pour les matériaux de la seconde classe (sables grossiers), il ne doit pas exister de lois intrinsicques. Les lois du transport hydraulique dans ce domaine doivent être une combinaison des lois ralables dans les deux classes voisines.

On roit aussi que, pour une étude sur modèle, on devra utiliser des malériaux appartenant à la mème classe que les matériaux de la nature. C'est pourquoi nous n'avons pu réduire l'échelle de notre modèle du circuit de refoulement de la drague en dessous du tiers, étant donné la granulométrie des déblais spécifiée au cahier des charges.

Remarquons enfin que, lorsque l'on change de fluide ou de matériaux, les diamètres critiques $d_{c}$ doivent satisfaire à la relation obtenue en écrivant que le nombre de Reynords $\frac{\mathrm{Wd}}{y}$ a une valeur constante $R_{c}$.

$$
\frac{g d_{c}^{3}}{v^{2}} \times\left(\frac{p^{\prime}-p}{p}\right) g=\text { Cste }
$$

Il nous était d'ailleurs difficile d'essayer de vérifier l'application de la similitude de Frovde pour les graviers et galets en faisant varier systématiquement l'échelle du modèle $\lambda$ en même temps que celle des matériaux. Nous avons dû nous contenter tout d'abord de faire varier le diamètre des grains dans la même installation de $104 \mathrm{~mm}$ de diamètre.

Nous pouvons penser que la valeur du rapport $\alpha=\frac{\mathrm{C}_{\mathrm{T}}}{\mathrm{C}_{\mathrm{s}}}$ de la concentration de transport à la concentration spatiale caractériscra l'écoulement et correspondra à une valeur de $\frac{V}{\sqrt{\lambda g}}$. Nous pouvons également considérer que les graviers en régime de saltation déerivent des trajectoires moyenmes types et possedent une hauteur d'ascension verticale $h$ pour chaque valeur de la vitesse moyenne et pour un diamètre de grain domné. Dans ces conditions, il peut sembler logique d'admettre que $\alpha$ est lié à la valeur de $\frac{h}{\mathrm{D}}$. Par suite, en combinant les deux conditions pricédentes, le paramètre $\frac{V}{\sqrt{\lambda g}}$ pour des matériaux à la même échelle que la conduite peut ètre remplacé par :

$$
\frac{\mathrm{V} \sqrt{d}}{g \mathrm{D}}
$$

et pour des matériaux différents dans la même conduite par :

$$
V \times \sqrt{d}
$$

Ceci peul sembler paradoxal. Pour conserver dans la conduite le même degré de transport, caractérisé par une valeur donnée de $\alpha$, il faut diminuer la vitesse si l'on augmente le diametre des graviers. C'est ce que nos observations nous avaient laissé pressentir et ce que nos mesures ont confirmé. En portant les valeurs expérimenlales de $\alpha$ en fonction de $V \times \sqrt{d}$, les points se groupent avee une assez bonne approximation autour d'une courbe unique (fig. 7). Les

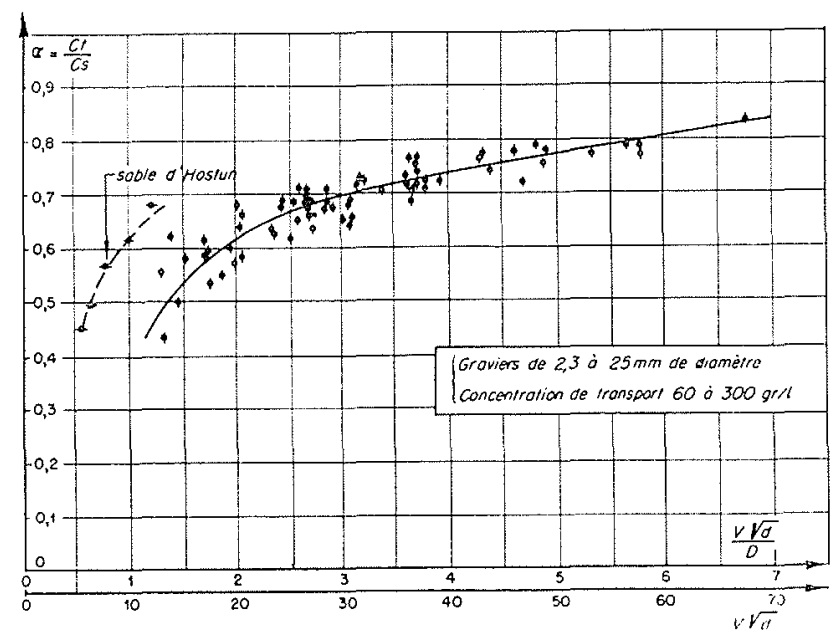

Fic. 7. - Variation de $\alpha$ en fonction de $(V \vee d)$ dans une conduite de $\varnothing 104 \mathrm{~mm}$.

joints correspondant à du sable grossier de $0,8 \mathrm{~mm}$ de diamètre moyen s'écartent nettement de cette courbe. Les régimes étudiés correspondent tous à des vitesses supérieures à la disparìtion de la « dune limite ». 
Nous avons igalement effectué des essais sur une conduite de $40 \mathrm{~mm}$ de diamètre et en prenant comme paramètre $\frac{V \times \sqrt{d}}{g \mathrm{D}}$ (fig. 8), les points se placent pour les deux modeles autour de la mème courbe. Le résultat est indépendant de la concentration dans la limite des essais (60 à $300 \mathrm{gm} / \mathrm{l})$.

Mais il est bien évident que de telles lois ne sont valables que dans la mesure où la vitesse

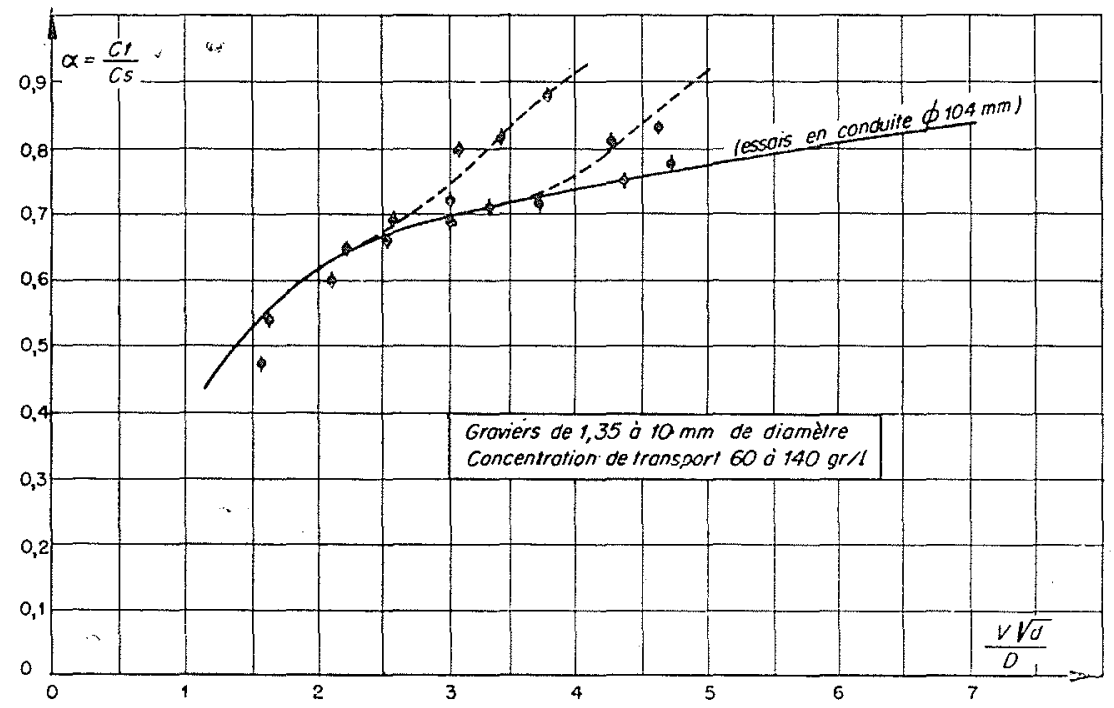

Fia. 8. - Variation de $\alpha$ en fonction de $\frac{V V \bar{d}}{D}$ dans une conduite de $\varnothing 41 \mathrm{~mm}$.

est suffisante pour entrainer les galets. Pour des valeurs de a roisines de zéro, correspondant au debut d'entrainement, on a en effet $\frac{V}{\sqrt{g d}}=$ Cste qui correspond toujours à Frovde, mais indépendamment cette fois, en première approximation, de l'échelle de la conduite.

Par ailleurs, lorsque la portée verticale moyenne des bonds tend à dépasser la valeur du diamètre de la conduite, les grains sont réfléchis dans tous les sens et la pesanteur tend à s'éliminer. On arrive ainsi à un pseudo-régime de suspension. Ce phénomène a pu également ètre mis en évidence au cours de nos essais, et correspond aux courbes en tirets de la figure 8 . Il est évidemment tout à fait particulier au cas des conduites en charge. Il ne présente sans doute pas d'intcirêt pour les rivières et canaux où une telle paroi réfléchissante n'existe pas et où les vitesses sont en outre généralement trop faibles.

Il résulte de ces remarques que nos résultats d'essais doivent être appliqués avec prudence aux installations à grande ćchelle refoulant des galets. Si nous avons pu dégager un aspect des lois générales du phénomène, les limites d'application demeurent encore incertaines.

En ce qui concerne les pertes de charge, nous n’avons pas vérifié la loi, classique pour les sa- bles fins, à savoir que la perte de charge est la même qu'en eau claire, à condition de l'exprimer en hauteur de mixture.

Pour les sables grossiers $(0,8 \mathrm{~mm})$ nous avons obtenu les résultats de la figure 9. Pour les gra-

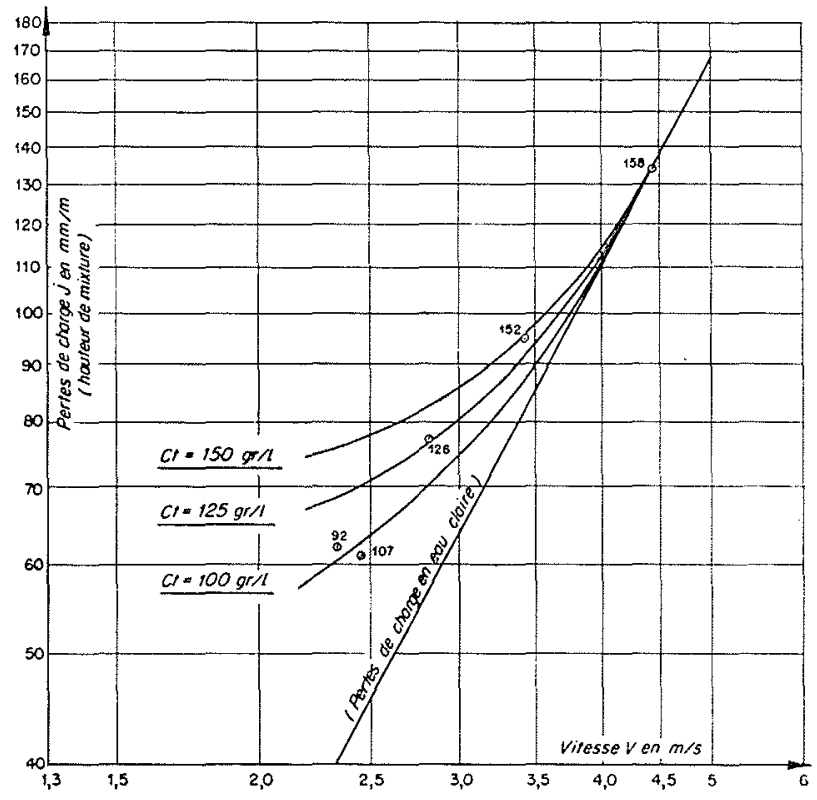

Fia, 9. - Pertes de charge pour du sable de $0,8 \mathrm{~mm}$ dans une conduite de $104 \mathrm{~mm}$ de diamètre. 
vicrs, nous donnons à titre d'exemple la figure 10 correspondant à un diamètre compris entre 2,3 et $5,25 \mathrm{~mm}$. Pour toutes les catégories de gra-

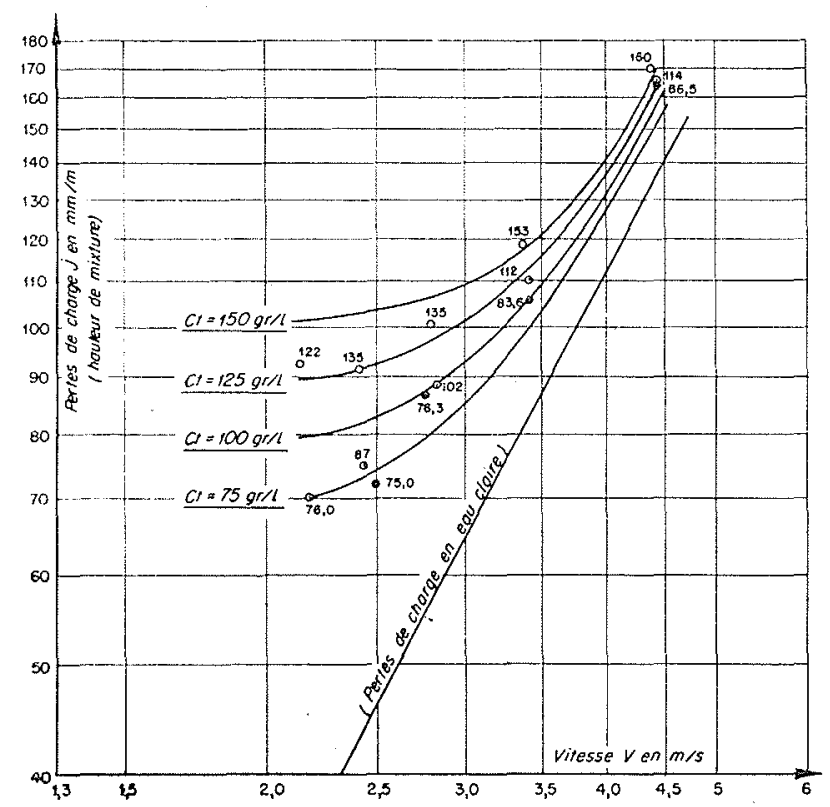

Fir. 10. - Pertes de charge pour des graviers de 2,3 à $5,25 \mathrm{~mm}$

dans une conduite de $104 \mathrm{~mm}$ de diamètre.

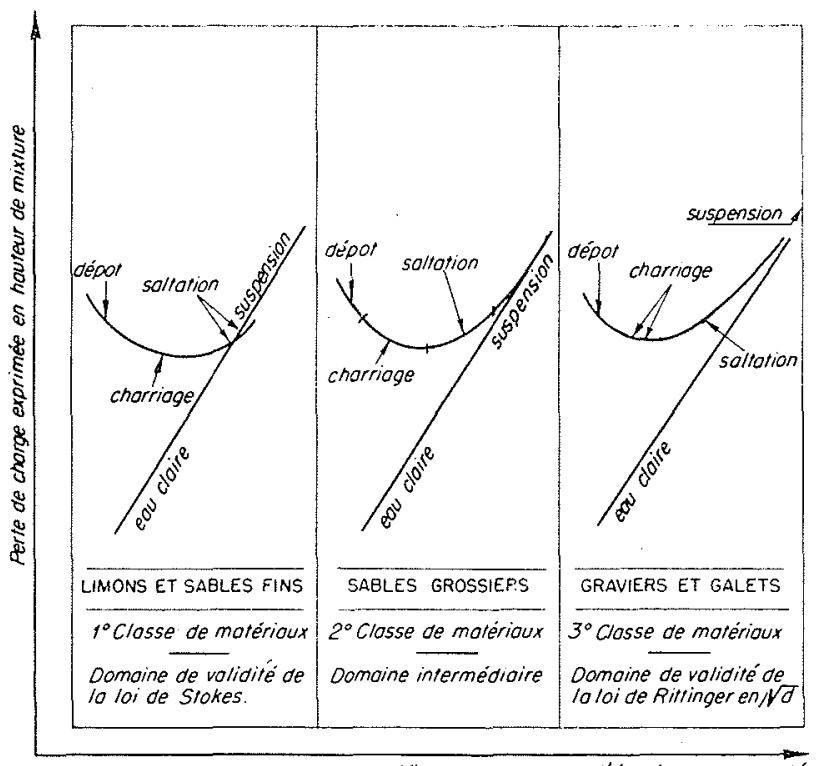

Vitesse moyenne de l'écoulement rapportée ò la section rotale de lo conduite.

FIG. 11. - Allure des lois de pertes de charge a concentration de transport constante pour les trois classes de matériaux (coordonnées logarithmiques).

viers étudiées, le coefficient de pertes de charge varie avec la concentration, mais semble indépendant du diametre moyen des grains.
L'allure des courbes de pertes de charge a concentration constante nous a conduits a la comparaison suivante schćmalisće par la figure 11 :

- En coordonnées logarithmiques pour les limons et sables fins, les courbes à $\mathrm{C}_{\mathrm{T}}$ constant se raccordent à la droite d'eau claire en formant avec cette dernière un angle assez net.

Pour de tels matériaux, le régime de saltation est pratiquement inexistant.

Pour les sables grossiers, les courbes rejoignent la droite de l'eau claire de facon plus progressive. Dans cette zone de transition entre le charriage et la suspension, la saltation joue déjà un rôle important.

- Pour les graviers el sans doute également pour les galels, les courbes semblent asymptotiques à la droite d'eau claire. Le phénomène de charriage tend à devenir secondaire et transitoire. Ia saltation est prépondérante et dans la gamme des vitesses réalisées qui sont déjà importantes, la suspension véritable n'apparait pas encore.

Il est assez normal que les pertes de charge soient plus fortes que pour des sables fins pour une même vitesse moyenne et que cet écart soit indépendant du diamètre des grains à partir d'une certaine limite. En effet, à chaque bond, le gravier dissipe de l'energie lorsqu'il vient heurter la conduite ou un autre gravier, du fait que le choc n'est pas parfaitement élastique. C'est l'écoulement qui doit lui restituer l'énergie perdue, ce qui se traduit finalement par une majoration des pertes de charge. Les matériaux fins, au contraire, ont une trop faible inertic et un trop grand coefficient de traine e pour pouvoir prendre, par rapport au fluide, des vitesses relativement importantes. Les chocs à la paroi sont donc négligeables pour eux. Pour les graviers, les choes répétés s'accompagnent d'un polissage parfait de la conduite (parfaitement lisse de Nikuradse) et d'une usure très rapide de galets, ce qui nous a d'ailleurs gênés dans la conduite des essais. On conçoit que la majoration des pertes de charge peut, en première approximation, n'être fonction que de la nature des choes et que, de ce fait, les pertes de charge peuvent varier avec la composition minéralogique des déblais et mème avec la nature de la conduite. Nous citerons à ce propos l'expérience curieuse suivante :

Des graviers isolés sont introduits dans la conduite vide de matériaux. Quel que soit le diamètre des graviers et la vitesse de l'écoulement (à condition qu'elle puisse entrainer le 
grain), la vitesse du grain par rapport à celle de l'eau reste de l'ordre de 0,96 dans la conduile de $\varnothing 104 \mathrm{~mm}$. Dans une conduite de $\varnothing 250 \mathrm{~mm}$, qui était restéc tapissée intérieurement de suies qui s'étaient tassées et restaient immobiles, prológées par le film laminaire, les mêmes graviers avaient une vilesse relative de 0,78 , également indépendante de leur diamètre. Ceci montre en outre que nos résultats ne sont plus valables pour de très fables concentrations et que la vitesse d'entrainement sur fond lisse est diffirente de celle sur fond rugueux.

II faut signaler également qu'une bille de verre ou d'acier isolée roule sans sauter sur le fond de la conduite, ce qui prouve qu'il est nécessaire que la forme des grains induise une portance sinon pour entretenir, du moins pour amorcer la saltation.

\section{CONCLUSION}

Celte étude, encore bien incomplete, montre cependant que la saltation est un mode de transport essentiel pour les materiaux qui possedent un coefficient de trainée constant en essai de vitesse de chute. La similitude du transport hydraulique semble alors devoir être celle de Frovde. Les résultats d'essais qui le prouvent justifient en même temps le classement des matériaux en trois domaines en fonction de la valeur du diametre des grains. Nous continuons celte étude plus spécialement dans le domaine intermédiaire des sables grossiers à l'oceasion de recherches en cours pour les Ponts et Chaussées de Nantes. Nous remercions les Ateliers et Chantiers de Bretagne d'avoir autorisé la communication de ces quelques résultats.

BIBLIOGRAPIILE

R. A. Bagnotb. - The Physies of Blown Sand and Desert Dunes. Bethuen (Londres).

-... British Journal of Applied Physics, vol, 2, p. 29-3t, revrier 1951.

Ch. Braxcher. -- La Honille Blanche, $\mathrm{n}^{\circ} 2$, mars 1946.

D) Boys. Annales des Ponts et Chaussées: Le Rhóne et les rivieres a fond affouillable. 1878.

Du Brat. - Principes d'Hydranlique, A l'imprimerie de Monsieur, 1786 .

Bnoryck. - Théorie du Lavage. Rente de l'Industrie Minérale, 1938 .

R. Domand. - Transport hychaulique de matériaux solides en conduite. La Honille Blanche, $\mathrm{n}^{\circ}$ 3, mai 1951.

P. Dunepare. - Contribution a l'etude du dragage et da refoulement des déblais a l'état de mixtule. Noureau type de pompes à debhais a Benciron-Ponts et Chanssers Annales des Ponts of Chansseses, 1939.
Mi. Escande - C.RA.S. 1949, page 613 , page 701 .

(. Haxoco. - Rente Uniberselle des Mines, févice 1928.

(i. Hanroxn. - Mesure des concentrations et des vitesses dans un contant mixle d'air of deau. La Honille Blanche, mai 1951.

(i. W. Howanb. -- ASCE, 1939, volume 104.

Sir C. Inasis. - The Behaviour and Control of Rivers and Canals. POONA 1949 .

Isbach. - Extrait des Annales de l'Institat Hydrotechnique de Recherches Scientifiques on U.R.S.S., volume XVII. 1935.

F. Hurstrom, - UPSALA, 1935, Bull, of the Geol. Instim tul, volume XXv.

Mrom-peten. Sichueizerische Banzeitung, 31 mars 1934.

M. P. O'Bnise et R. G. Forson. - The Transportation of Sund in pipe lines. Universite de Californie, 12 novembre 1937.

\section{I S C U S I O N}

M. le Président remercie M. Robert Dunavo et insiste sur les points suivants :

La communication porte sur un certain nombre d'observations of de résultats tiés des essais systématiques actuellement en cours sur les ecoulements de mixtures en conduites. Ces essais portent sur des mixtures ou tous les facleurs sont appeies à varier, grosseur et nature des grans, densite, concentration, vitesse, diametre des conduites.

Dans l'exposé qui vient d'être fait il y a lieu d'attires lattention sur la notion originale du coefficient a rappost entre la concentiation de transport et la concentration spatiale, étudié ensuite en fonction de la vitesse dans la conduite.

Cette représentation met en évidence d'une façon remarquable le comportement physique de chaque écoulement considér. Permettant de ce fait l'analyse des phénomenes elle doit faciliter la mise a point des lois de similitudes éventuelles.

M. Dunand vient d'en donner une preuve immédiate en envisageant lexistence probable d'une loi unique de $a$ en fonction de $\frac{\sqrt{d}}{\sqrt{l}}$ lorsque les conditions sont telles que les effets de la viscosité deviennent négligeables.
M. le président félicite M. Denaxd de ce premier risultat en espérant qu'il conlintera à mettre méthodiquement de la clarté dans ees phénomènes rendus complexes par le grand nombre de variables.

II. Schlag signale que les essals de M. Gubcony ont élé analysés par M. Hanoco, professeur à l'Université de Liege, qui les a traduits dans la representation graphicue classique : $\lambda=f($ R)

(Rappelons que ces essais ont ete effectues sur nne conduite de $4^{\prime \prime}$ de diamètre, avec une cau contenant de 0 à $35 \%$ en poids de matériaux solides très fins, en suspension).

Les conclusions de M. Haxoce peuvent se résumer comme suit :

On peut distinguer les deux régimes, laminaire et turbulent, mais la valeur du nombre critique du nombre de Reynolds qui les sépare croît avec la teneur de matières en suspension; il semble donc que la présence de particules solides tende à maintenir plus longtemps le régime laminaire.

Pour l'écoulement turbulent, le $\lambda$ correspond à celui des tuyaux lisses, comme s'il se produisait sur la paroi un mince dépôt de matière transformant la paroì rugueuse en paroi lisse. 